\title{
UTILIZAÇÃO DO PERFIL ISOENZIMÁTICO COMO FERRAMENTA NA AVALIAÇÃ O DA SELETIVIDADE DE HERBICIDAS EM CANA-DE-AÇÚCAR
}

\author{
BELUCI, Lucas Ribeiro ${ }^{1}$ \\ VITORINO, Renan ${ }^{2}$ \\ AZANIA, Carlos Alberto Mathias ${ }^{2}$ \\ AZANIA, Andrea de Padua Aparecida Mathias \\ TORTORELLI, Henrique Freitas
}

RESUMO: Um herbicida pode ser seletivo quando o perfil isoenzimático das enzimas $\alpha$-esterase e peroxidase nas plantas tratadas for similar ao das plantas testemunhas. Objetivou-se estudar a aplicabilidade do perfil isoenzimático da $\alpha$-esterase e peroxidase, comparativamente às características fitotécnicas, como ferramenta para avaliar a seletividade na cultura da cana-de-açúcar, utilizando diversos herbicidas registrados em pré-plantio. A pesquisa foi realizada em duas etapas, inicialmente em vasos, alocados em ambiente aberto e depois em laboratório. O delineamento experimental foi inteiramente casualisado com 8 tratamentos [testemunha; imazapic (245 $\left.\mathrm{g} \mathrm{ha}-{ }^{-1}\right)$; diclosulam $\left(88,2 \mathrm{~g} \mathrm{há}^{-1}\right)+\mathrm{s}$-metolachlor $\left(1920 \mathrm{~g} \mathrm{ha}^{-1}\right)$; imazapyr (500 $\left.\mathrm{g} \mathrm{ha}^{-1}\right)$; diclosulam (88,2 $\left.\mathrm{g} \mathrm{ha}^{-1}\right)$ +oxyfluorfen (1200 $\left.\mathrm{g} \mathrm{ha}^{-1}\right)$; amicarbazone (2100 $\left.\mathrm{g} \mathrm{ha}^{-1}\right)$; trifluralin $\left(4240 \mathrm{~g} \mathrm{ha}^{-1}\right)+$ pendimethalin $\left(1750 \mathrm{~g} \mathrm{ha}^{-1}\right)$; sulfentrazone (800 $\mathrm{g}$ ha- $\left.{ }^{1}\right)$ ] em 4 repetições e em 3 épocas de plantio, considerando o período a partir da aplicação dos tratamentos no solo. A primeira, segunda e terceira época de plantio foram, respectivamente, 20, 40 e 60 dias após a aplicação dos tratamentos (DAA). As unidades experimentais foram constituídas por vasos de plástico (3L) preenchidos com solo de barranco. O herbicida foi aplicado com pulverizador costal pressurizado, munido de barra com quatro pontas jato leque (TT110/02), espaçadas de $0,50 \mathrm{~m}$, pressão constante de $2,1 \mathrm{kgf} \mathrm{cm}^{-2} \mathrm{e}$ volume de calda de $200 \mathrm{~L} \mathrm{ha}^{-1}$. Após 20 , 40 e 60 DAA, simultaneamente foi plantado 1 MPB (muda pré-brotada) de cana de açúcar por parcela. As avaliações visuais de sintomas foram feitas aos 20, 40 e 60 dias após o plantio (DAP) e aos, 60 DAP foram feitas as avaliações do teor de clorofila, altura de plantas, massa seca e coleta de material para caracterização do perfil enzimático. Verificou-se semelhanças refletindo na intensidade das isoformas $\alpha$-esterase e peroxidase nos mesmos tratamentos, a partir da metodologia para quantificar o fenômeno da seletividade Todavia, a hipótese inicial foi confirmada devido ao padrão das isoformas $\alpha$-esterase e peroxidase não diferirem em número de bandas, corroborando com as características fitotécnicas,a partir da metodologia feita para quantificar a seletividade. Dessa forma verificou-se seletividade das MPBs de cana da cultivar IACSP95-5000, permitindo a utilização do perfil isoenzimático como uma ferramenta para avaliação de seletividade de herbicidas quando associado às avaliações fitotécnicas.

Palavras-chave: $\alpha$-esterase. Peroxidase. MPB.

SUMMARY: A herbicide may be selective when isoenzyme profile of $\alpha$-esterase and peroxidase enzymes in treated plants is similar to that of control plants. The objective was to study the applicability of the isoenzyme profile of $\alpha$-esterase and peroxidase compared the phytotechnical characteristics as a tool to assess the selectivity in sugarcane using various herbicides registered for pre-planting. The research carried out in two steps in pots in an open environment and in the laboratory. The experimental design was completely randomized with eight treatments [Control; imazapic (245 $\left.\mathrm{g} \mathrm{ha}^{-1}\right)$; diclosulan $\left(88,2 \mathrm{~g} \mathrm{ha}^{-1}\right)+\mathrm{s}$-metalachlor $\left(1920 \mathrm{~g} \mathrm{ha}^{-1}\right)$; imazapyr $\left(500 \mathrm{~g} \mathrm{ha}^{-1}\right)$; diclosulan $\left(88,2 \mathrm{~g} \mathrm{ha}^{-1}\right)$ +oxyfluorfen (1200 $\left.\mathrm{g} \mathrm{ha}^{-1}\right)$; amicarbazone (2100 $\mathrm{g}$ ha-1); trifluralin $\left(4240 \mathrm{~g} \mathrm{ha}^{-1}\right)+$ pendimethalin (1750 $\mathrm{g}$ ha- $\left.{ }^{1}\right)$; sulfentrazone $\left.\left(800 \mathrm{~g} \mathrm{ha}^{-1}\right)\right]$ in four replicates and three planting dates defined planting days after application (20DAA, 40DAA e 60DAA). The experimental units consisted of plastic pots (3L) filled with soil. The herbicide was applied by spraying pressurized bar with four jet tips range (TT110/02), spaced $0.50 \mathrm{~m}$, constant pressure $2,1 \mathrm{kgf}$ $\mathrm{cm}^{-2}$ and spray volume of $200 \mathrm{~L} \mathrm{ha}^{-1}$. After 20, 40 e 60 DAA were planted simultaneously 1 MPB (One Eye Set) sugarcane per plot. The variables phytotechnical evaluated were, intoxication visual symptoms (scale 0 a $100 \%$ ), chlorophyll content (units) plant height (considering the soil down to the hem of the last fully developed leaf) and dry weight 60 days after planting (DAP). At 60 DAP characterized isoenzyme profile of $\alpha$-esterase and peroxidase in the laboratory, by electrophoresis on acrylamide gel and were made the zimograms in Excel 2010 software. However, the initial hypothesis was confirmed due to the pattern of $\alpha$-esterase and peroxidase isoforms did not differ in number of bands, corroborating the phyto technical characteristics. Thus there was selectivity of sugarcane cultivar IACSP 95-5000 MPBs (One Eyes Sets), allowing the use of isoenzyme profiles as a tool to herbicide

\footnotetext{
${ }^{1}$ FCAV UNESP JABOTICABAL -

${ }^{2}$ IAC - Centro de Cana - Ribeirão Preto
} 
selectivity assessment when associated with phytotechnical pattern.

Keywords: $\alpha$-esterase. Peroxidase. One Eye Set.

\section{INTRODUÇÃO}

A seletividade é a capacidade dos herbicidas em eliminar as plantas daninhas sem reduzir a produtividade e a qualidade do produto final obtido no cultivo (ABU-QARE; DUNCAN, 2002; NEGRISOLI, et al. 2004). Também é relacionada à capacidade da cultura em absorver e metabolizar os herbicidas, sendo essa uma característica intrínseca a cada cultura e suas cultivares. O herbicida deixa de ser seletivo quando não ocorre a desativação metabólica de sua molécula no metabolismo da planta, que passa ser susceptível e morre (BREAUX, 1987).

Os herbicidas modernos alicerçam-se na inibição das atividades enzimáticas, seja da síntese de aminoácidos, proteínas, lipídeos, pigmentos fotossensíveis ou ainda hormônios. Assim, desencadeiam uma série de distúrbios celulares que levam as plantas susceptíveis à morte (MACHADO et al. 2006).

Uma vez que os herbicidas atingem as células vegetais, a capacidade de defesa das plantas é ativada. Entre os complexos mecanismos de defesa, a planta dispõe das enzimas de desintoxicação, que catalisam a conjugação da enzima glutationa com os mais diferentes substratos citotóxicos como xenobióticos (BILANG et al., 1993). Nas células, a presença da molécula de herbicida causa estresse oxidativo que gera radicais livres, peróxidos e superóxidos. O alívio desse estresse é realizado por enzimas, como a $\alpha$-esterase e peroxidase.

O sistema isoenzimático $\alpha$-esterase é constituído por um complexo e heterogêneo grupo de enzimas reativas com uma ampla gama de substratos específicos (SCANDÁLIOS,1969). Estas, por sua vez, possuem a propriedade de catalisar a hidrólise de ésteres, peptídeos, amidas e haletos (WALKER;MACKNESS, 1983).

A peroxidase é fundamental no metabolismo das plantas, utiliza-se de peróxidos como aceptor de hidrogênio, podendo colaborar no aumento dos mecanismos de defesa e prevenção de perda da qualidade (USHIMARU et al., 2001). Segundo Bewley; Black (1994), a diminuição da atividade enzimática concede maior exposição dos sistemas de membranas aos efeitos do oxigênio. Assim, em decorrência do gradiente de danos das membranas, o oxigênio atua de forma mais acentuada ocasionando oxidação dos compostos e degradação de lipídeos.

Plantas com altas taxas de antioxidantes, constitutivos ou induzidos, são reportadas como mais tolerantes a danos oxidativos, dessa forma induz mudanças nos padrões dos sistemas isoenzimáticos da peroxidase e são constantes em plantas sujeitas a estresses abióticos (DIONÍSIO-SESE; TOBITA, 1998; SREENIVASULU et al., 1999; ULISSES et al., 2002).

Além disso, a peroxidase tem inúmeras e importantes funções vitais, como ajuste osmótico, produção de NADPH, regulação iônica, participa da fotorrespiração e atua na fixação de $\mathrm{CO}_{2}$ sendo cada função dependente da presença em determinada organela distinta (ALFENAS et al., 2006).

A atividade enzimática da $\alpha$-esterase e peroxidase é fundamental para manter ou diminuir os níveis de radicais $\mathrm{O}_{2} \mathrm{e}_{2} \mathrm{O}_{2}$, ao sequestrar íons metálicos e assim minimizar a formação do radical $\mathrm{OH}$, que é tóxico para as células, pois degradam lipídeos de membrana (MITTLER, 2002).

Assim levantou-se como hipótese que um herbicida possa ser seletivo quando o perfil isoenzimático das enzimas $\alpha$-esterase e peroxidase nas plantas tratadas for similar ao das plantas 
testemunhas. Para comprovar a hipótese objetiva-se estudar a aplicabilidade do perfil isoenzimático da $\alpha$ esterase e peroxidase, comparativamente às características fitotécnicas, como ferramenta para avaliar a seletividade dos diferentes herbicidas aplicados em pré-plantio na cultura da cana-de-açúcar.

\section{MATERIAL E MÉTODO}

O experimento foi realizado a $21^{\circ} 12^{\prime} 28.29^{\prime \prime}$ de latitude Sul, $47^{\circ} 52^{\prime} 23.30^{\prime \prime}$ de longitude Oeste e a $621 \mathrm{~m}$ de altitude. O local possui clima característico de verões quentes e úmidos e invernos secos e frios, considerado como tropical de altitude (Cwa), segundo a classificação de Köppen. O experimento foi realizado em duas etapas, inicialmente em vasos, alocados em ambiente aberto e depois em laboratório.

Na primeira etapa, o experimento foi conduzido no período de setembro de 2013 a janeiro de 2014, em vasos de plástico (3L), que constituíram as unidades experimentais, preenchidos com terra de barranco de textura argilosa e alocados em ambiente aberto.

O substrato utilizado foi solo de textura argilosa $(58,1 \%$ de argila, $16,8 \%$ de areia e $25,1 \%$ de silte), proveniente de barranco. A composição química do solo apresentava $6,6 \mathrm{de} \mathrm{pH}\left(\mathrm{CaCl}_{2}\right) ; 8 \mathrm{~g} \mathrm{dm}^{-3} \mathrm{de}$ matéria orgânica; 71,96\% de V\%; $3 \mathrm{mg} \mathrm{dm}^{-3}$ de $\mathrm{P}_{\text {(resina) }}$ e 0,$74 ; 30,4 ; 4,78 ; 14 \mathrm{mmol}_{\mathrm{c}} \mathrm{dm}^{-3}$, respectivamente, de $\mathrm{K}, \mathrm{Ca}, \mathrm{Mg}$ e $\mathrm{H}+\mathrm{Al}$. A terra foi peneirada, para eliminar material vegetal e torrões.

Os herbicidas (Tabela 1) foram aplicados com pulverizador costal pressurizado com $\mathrm{CO}_{2}$, regulado a 30 psi de pressão e equipado com pontas TT110/02 proporcionando volume de calda de $200 \mathrm{~L}$ $\mathrm{ha}^{-1}$. Na sequencia, os vasos foram distribuídos no local em delineamento inteiramente casualizado com os 8 tratamentos em 4 repetições, para cada época de plantio (20, 40 e 60 DAA). A aplicação ocorreu em $18 / 10 / 2013$ com início às $15: 45 \mathrm{~h}$, ocasião em que constatou-se $24,6^{\circ} \mathrm{C}$ de temperatura do ar, $67 \%$ de umidade relativa do ar, velocidade do vento de $6,5 \mathrm{~km} \mathrm{~h}^{-1}$ e nebulosidade de $40 \%$. O término deu-se às 16:10 h à temperatura de $28,4^{\circ} \mathrm{C}$, umidade relativa do ar $60 \%$, velocidade do vento de $9 \mathrm{~km} \mathrm{~h}^{-1}, 50 \%$ de nebulosidade.

Transcorridos 20, 40 e 60 DAA (dias após a aplicação) respectivamente, foi plantada uma muda pré-brotada (MPB) de cana-de-açúcar da cultivar IACSP95-5000 em cada vaso, respeitando cada época de plantio (20, 40 e 60 DAA), de modo que haviam 3 épocas de plantio, para cada tratamento e testemunha.

Tabela 1. Tratamentos utilizados nos experimentos com MPB (cultivar IACSP95-5000). Ribeirão Preto, 2014.

\begin{tabular}{|c|c|c|}
\hline Tratamentos & Nome comercial & Ingrediente ativo \\
\hline $\mathrm{T} 1$ & testemunha & Testemunha \\
\hline $\mathrm{T} 2$ & Plateau (350 $\left.\mathrm{g} \mathrm{ha}^{-1}\right)$ & imazapique (245 $\left.\mathrm{g} \mathrm{ha}^{-1}\right)$ \\
\hline $\mathrm{T} 3$ & Coact $\left(105 \mathrm{~g} \mathrm{ha}^{-1}\right)+\operatorname{dual}\left(2 \mathrm{~L} \mathrm{ha}^{-1}\right)$ & $\operatorname{diclosulam}\left(88,2 \mathrm{~g} \mathrm{ha}^{-1}\right)+\mathrm{s}-$ metalacloro $\left(1920 \mathrm{~g} \mathrm{ha}^{-1}\right)$ \\
\hline $\mathrm{T} 4$ & Contain $\left(2000 \mathrm{~mL} \mathrm{ha}^{-1}\right)$ & imazapir (500 g ha- $\left.{ }^{1}\right)$ \\
\hline T5 & Coact $\left(105 \mathrm{~g} \mathrm{ha}^{-1}\right)+\operatorname{Goal}\left(5 \mathrm{~L} \mathrm{ha}^{-1}\right)$ & diclosulam $\left(88,2 \mathrm{~g} \mathrm{ha}^{-1}\right)$ +oxyfluorfen $\left(1200 \mathrm{~g} \mathrm{ha}^{-1}\right)$ \\
\hline T6 & Dinamic $\left(3000 \mathrm{~g} \mathrm{ha}^{-1}\right)$ & amicarbazona $\left(2100 \mathrm{~g} \mathrm{ha}^{-1}\right)$ \\
\hline $\mathrm{T} 7$ & Trifluralina Nortox Gold $\left(5000 \mathrm{~mL} \mathrm{ha}^{-1}\right)+$ Herbadox & trifluralin $\left(4240 \mathrm{~g} \mathrm{ha}^{-1}\right)+$ pendimethalin $\left(1750 \mathrm{~g} \mathrm{ha}^{-1}\right)$ \\
\hline $\mathrm{T} 8$ & $\operatorname{Boral}\left(1600 \mathrm{~mL} \mathrm{ha}^{-1}\right)$ & sulfentrazona $\left(800 \mathrm{~g} \mathrm{ha}^{-1}\right)$ \\
\hline
\end{tabular}

A seletividade dos herbicidas foi avaliada de acordo com os sintomas das plantas aos 20, 40 e 60 dias após plantio (DAP). Avaliou-se visualmente, os sintomas de intoxicação na parte aérea das plantas, utilizando-se da escala percentual de notas, onde 0 representava a ausência de sintomas e 100 a morte das plantas. O teor de clorofila foi obtido por três leituras no terço médio da folha +1 , utilizando-se do 
medidor de clorofila, modelo SPAD 502 do fabricante Minolta. A altura das plantas $(\mathrm{cm})$ também foi avaliada, medindo a distância do solo até a aurícula da última folha completamente desenvolvida.

Aos 60 DAP, foi obtida a massa seca das plantas. Primeiramente, as plantas de cada parcela foram cortadas rente ao solo, colocadas em saquinhos de papel e mantidos em estufa de circulação forçada a ar $\left(70^{\circ} \mathrm{C}\right)$ até peso constante.

$\mathrm{Na}$ segunda etapa do experimento, aos 60 DAP foram coletadas folhas para avaliar o perfil isoenzimático das enzimas peroxidase e $\alpha$-esterase em laboratório.

Na ocasião da coleta, em cada tratamento (Tabela 1), foi retirada uma folha de cada repetição, da qual foi considerado $5 \mathrm{~cm}$ do limbo foliar do terço médio para constituir a amostra composta que, por sua vez, foi acondicionada em cassetes de plástico do tipo histológico.

Estes foram mantidos em caixa de isopor contendo gelo e posteriormente transportados ao laboratório e armazenados em freezer $-20^{\circ} \mathrm{C}$ até o processamento.

No momento da extração, para cada amostra, foram pesados $0,2 \mathrm{~g}$ de tecido foliar, macerados em cadinho de porcelana com auxílio de nitrogênio líquido. Segundo Alfenas et al. (2006), um dos maiores problemas encontrados durante a extração para estudos de isoenzimas é a liberação, nos tecidos vegetais durante a maceração, de compostos secundários, principalmente compostos fenólicos que reagem com proteínas e inativam enzimas ou alteram sua mobilidade no gel. Para minimizar os efeitos destes compostos, foram adicionados 0,04 g de PVP 10 (polivinilpirrolidona) a cada amostra macerada. Este composto tem a capacidade de adsorver e inativar fenóis. Além disso, adicionou-se $0,8 \mathrm{~mL}$ de solução tampão de extração contendo antioxidantes e agentes quelantes [0,6 g de fosfato de sódio bibásico; $7 \mathrm{~g}$ de sacarose; 2,56 g de PVP-10; $100 \mathrm{mg}$ de L-ácido ascórbico; $50 \mathrm{mg}$ de bissulfito de sódio; $50 \mathrm{mg}$ de borato de sódio; 0,2 $\mathrm{mL}$ de $\beta$-mercaptoetanol; $1 \mathrm{~g}$ de polietilenoglicol; $100 \mathrm{~mL}$ de água deionizada, adaptado de Alfenas et al. (2006). Em seguida, o extrato obtido foi centrifugado por três minutos a $1500 \mathrm{rpm}$ sendo o sobrenadante transferido para um microtubo e armazenado a $-20{ }^{\circ} \mathrm{C}$.

Todo o procedimento de extração, e posteriormente a eletroforese, foi realizado em baixas temperaturas e as amostras mantidas sob gelo em tempo constante. A extração foi realizada em tempo mínimo para minimizar possíveis problemas de proteólise citado por Almeida \& Crócomo (1994), no qual, poderia promover degradação de proteínas.

Para a separação das isoenzimas optou-se pelo uso de poliacrilamida-Bis pelo seu maior poder de resolução, devido à ampla variação controlável no diâmetro de seus poros.

As isoformas tanto da peroxidase como da $\alpha$-esterase foram separadas em um sistema vertical de eletroforese em gel de Acrilamida-bis (30:0,8\%) não desnaturante contendo tampão de corrida Tris Glicina $(0,05 \mathrm{M}, \mathrm{pH} 8,0)$. Para a montagem do gel de poliacrilamida foram utilizados gel separador a $7 \%$ e concentrador a $5 \%$. Para a separação foram utilizados uma alíquota de $30 \mu \mathrm{L}$ do extrato de tecido foliar. A eletroforese foi conduzida em geladeira a $4^{\circ} \mathrm{C}$ para evitar a desnaturação das enzimas, a 200 volts por aproximadamente dez horas (ALFENAS; BRUNE, 2006a).

Após a corrida, os géis foram incubados em solução de coloração. Para coloração do sistema enzimático $\alpha$-esterase, inicialmente os géis foram mantidos recipiente de vidro contendo $100 \mathrm{~mL}$ de solução tampão fosfato $0,1 \mathrm{M} \mathrm{pH} \mathrm{6,2} \mathrm{[81,5} \mathrm{mL} \mathrm{de} \mathrm{solução} \mathrm{A:} \mathrm{NaH}_{2} \mathrm{PO}_{4} \cdot 1 \mathrm{H}_{2} \mathrm{O}$ 0,1M (4,14 g / $\left.300 \mathrm{~mL}\right)+$ $18,5 \mathrm{~mL}$ de solução B: $\mathrm{Na}_{2} \mathrm{HPO}_{4}$ 0,1 M (4,26 g / $\left.300 \mathrm{~mL}\right)$ ] em agitador tipo "Rocking Platform" Bio Rad a $20 \mathrm{rpm}$. Em seguida foram adicionados os reagentes $\alpha$-naftil acetato e Fast Blue RR. Primeiro foram dissolvidos $0,02 \mathrm{~g}$ de $\alpha$-naftil acetato em $500 \mu \mathrm{L}$ de acetona, em seguida esta solução dissolvida em $80 \mathrm{~mL}$ de solução tampão fosfato. Após um período de 15 minutos foram adicionados $20 \mathrm{~mL}$ da mesma solução 
tampão fosfato contendo 0,018 g de Fast Blue RR em condição de escuro, até o aparecimento de bandas (adaptado de ALFENAS; BRUNE et al., 2006a).

Para o sistema enzimático peroxidase, o gel foi mantido em recipiente de vidro por 1 hora contendo o fixador acetato de sódio 0,10M pH 5,0 em agitador tipo "Rocking Platform" Bio Rad a 20 rpm. Após esse período, a solução fixadora foi retirada e adicionadas as soluções 1 (100 mg de 3-amino-9ethyl dissolvidos em 2,5 mL de N,N-dimetil-formamida) e 2 (60 mL de tampão peroxidase - acetato de sódio $0,05 \mathrm{M}$ pH 5,0; $3 \mathrm{~mL}$ de $\mathrm{CaCl}_{2}$,, $10 \mathrm{M}$ e $200 \mu \mathrm{l}$ de $\mathrm{H}_{2} \mathrm{O}_{2}$ ) mantidas durante aproximadamente 20 minutos a 1 hora, no escuro, para revelação das bandas. Decorrido esse tempo, a solução foi descartada, o gel lavado com água e adicionada à solução descorante por 10 minutos. Em seguida, foi adicionada a solução secadora.

No final de cada processo de coloração, os géis foram incubados em solução secadora (ALFENAS;BRUNE, 2006b) "over night" e no dia seguinte, cada gel foi envolvido com papel celofane previamente umedecido com solução secadora, submetidos ao equipamento de secagem forçada "Gel Dryer Model 583” Bio Rad na configuração PAGE (Polyacrylamide Gel Electrophoresis) por 40 minutos a $80{ }^{\circ} \mathrm{C}$.

A leitura das isoenzimas foi realizada com auxílio de um transiluminador, onde os perfis isoenzimáticos dos tratamentos foram comparados aos perfis das respectivas testemunhas em cada época de avaliação. Utilizando-se do software Excel, reproduziram-se as bandas e suas intensidades, confeccionando o zimograma.

Os dados obtidos no campo foram submetidos à análise de variância pelo teste $\mathrm{F}$ e as médias comparadas pelo teste de Tukey a 1 e $5 \%$ de probabilidade.

\section{RESULTADOS E DISCUSSÃO}

Os sintomas de intoxicação ocorreram apenas na época 1 (20DAA) e persistiram até aos 40 DAP. $\mathrm{O}$ tratamento que teve os maiores sintomas foi o T4 (imazapir 500 $\mathrm{g} \mathrm{ha}^{-1}$ ), com $26 \%$ aos 20 DAP, diminuindo para 9,75\% aos 40 DAP e igualando-se à testemunha aos 60 DAP (Tabela 2), acusando uma possível recuperação. Nas épocas 2 (40DAA) e 3 (60DAA), os tratamentos não tiveram quaisquer sintomas observados. Beluci et al. (2013) observaram que para imazapir 500g ha ${ }^{-1}$, também em MPBs de cana de açúcar, aos 83 (30 DAP), 113 (60DAP) e 133 (80DAP) dias após a aplicação, respectivamente, ocorreram sintomas de $47 \%, 63 \%$ e $44 \%$.

Para o teor de clorofila, não ocorreram diferenças significativas em relação à testemunha nas 3 épocas até os 60 DAP. Uma vez que, de acordo com Lopes et al. (2009), a clorofila se relaciona com a eficiência do processo de fotossíntese e a mínima interferência no processo afeta o desenvolvimento da planta, reforça a possibilidade de que mesmo ocorrendo sintomas leves de intoxicação na época 1, estes não foram suficientes para prejudicar o teor de clorofila constatado nas avaliações.

Observando as épocas 2 e 3, nenhuma significância estatística foi verificada (Tabela 2). Corroborando com os dados, resultados similares foram verificados por Vitorino (2013), trabalhando com MPBs de cana e utilizando doses de amicarbazone (de 1440 até $4200 \mathrm{~g} \mathrm{ha}^{-1}$ ) e imazapir (de 500 até $1500 \mathrm{~g} \mathrm{ha}^{-1}$ ). O autor observou em pré-emergência 43,08 unidades relativas de clorofila aos 60DAP (165 DAA) na testemunha e não ocorreram diferenças significativas em relação ao teor de clorofila para as doses destes herbicidas. 
A altura das plantas apresentava em média nas testemunhas 11,6; 14,3 e 12,9 cm respectivamente, aos 20, 40 e 60 DAP, porém, não diferiram dos tratamentos herbicidas indicando que mesmo as plantas estarem gastando energia metabolizando herbicidas, em alguns tratamentos, o gasto não foi suficiente para manifestar quedas no crescimento das plantas. Silva et al. (2013) não observaram expressivas alterações na altura das plantas até os 58 DAA utilizando herbicidas de diferentes mecanismos de ação, entre eles o sulfentrazona (800 $\left.\mathrm{g} \mathrm{ha}^{-1}\right)$, nas cultivares IACSP95-5094 e IACSP94-2042.

A massa seca foi semelhante ao teor de clorofila e a altura das plantas. As testemunhas aos 60 DAA apresentaram valores de 6,41 $\mathrm{g}$ planta $^{-1}$ em média, não sendo suficiente para diferir dos tratamentos herbicidas. Isto evidencia ainda mais a possibilidade de que a cultivar utilizada (IACSP95-5000) na forma de MPB, foi tolerante aos herbicidas utilizados no estudo, por não prejudicarem o teor de clorofila, altura e principalmente a massa seca. Beluci et al. (2013) verificaram que amicarbazona e imazapir nas doses recomendadas pelo MAPA, para a cultivar IACSP95-5000, não interferiram nas variáveis fitotécnicas, entre elas a massa seca até os 53 DAA, assim, a cultivar demonstrou tolerância a estes herbicidas.

Observando-se o zimograma aos 60 DAP (Tabela 3), no perfil isoenzimático da peroxidase não houveram diferenças quanto ao número de bandas nas 3 épocas, indicando que ...... Em relação à intensidade das bandas, com 20 DAA na época 1, os tratamentos T2, T4, T6 e T7 tiveram as bandas um pouco mais evidenciadas do que a testemunha, enquanto que aos 40 DAA na época 2, somente T6, T7 e T8 foram diferentes em intensidade. Aos $60 \mathrm{DAA}$, na época 3, todos os tratamentos mantinham o mesmo padrão da testemunha, indicando que as isoformas da peroxidase tiveram similaridade à testemunha.

Silva et al (2013), estudando interações entre diferentes níveis de adubação e herbicidas de diferentes mecanismos de ação, em pós emergência da cana, obtiveram diferenças em número de bandas para peroxidase, porém não verificaram essas diferenças para a $\alpha$-esterase. Beluci et al (2014), relataram que o perfil da $\alpha$-esterase foi alterado em número quando utilizou-se de doses de glyphosate (de 1440 a $4320 \mathrm{~g} \mathrm{ha}^{-1}$ ) 1 e 12 dias respectivamente antes do plantio de Glycine max e Zea mays, mas não encontraram estas diferenças para Arachis hypogaea. Adriano et al. (2013) constataram que o perfil de $\alpha$ esterase apresentou comportamentos diferentes estudando 3 cultivares de cana-de-açúcar (IACSP94-4004, RB724554 e IACSP93-3046) submetidos a doses de glyphosate (de 1440 a $4320 \mathrm{~g} \mathrm{ha}^{-1}$ ) em pós emergência, assim, verificou que somente a cultivar IACSP94-4004 diferiu em número de bandas em relação à testemunha.

Em relação a intensidade das bandas, na época 1, os tratamentos T2, T4, T6 e T7 propiciaram maior intensidade para peroxidase e T2, T4, T6, T7 e T8 para $\alpha$-esterase. Para a época 2, os tratamentos T6, T7 e T8 tiveram maior intensidade para peroxidase e T2, T6, T7 e T8 para $\alpha$-esterase. Desse modo, mesmo esperando 40 dias após a aplicação dos herbicidas para o plantio da cana, estes de herbicidas foram absorvidos pelas plantas como indicam as atividades enzimáticas para metabolização. . Na época 3 não ocorreram alterações no perfil da peroxidase em relação à testemunha, porém, os tratamentos T6 e T7 no perfil de $\alpha$-esterase ainda apresentavam maior

intensidade nas bandas, dessa forma, T6 e T7 apresentaram maior intensidade nas bandas para três épocas. Silva et al (2013) averiguaram que a intensidade das isoformas de $\alpha$-esterase e peroxidase diferiu para tratamentos herbicida, o que pode ser um indício de maior atividade enzimática, principalmente pela $\alpha$-esterase, que mesmo sem aparentar sintomas de intoxicação aos 58DAA apresentou menor intensidade nas bandas e ainda estava em processo de metabolização dos herbicidas. 
Tabela 2. Avaliações fitotécnicas de seletividade em três épocas de plantio após aplicação de herbicidas.

\begin{tabular}{|c|c|c|c|c|c|c|c|c|c|c|c|c|c|c|c|c|c|c|c|c|}
\hline \multirow{3}{*}{ 兽 } & \multirow{3}{*}{ Tratamentos } & \multicolumn{5}{|c|}{ sintomas de intoxicação (\%) } & \multicolumn{6}{|c|}{ teor de clorofila (unidades relativas) } & \multicolumn{6}{|c|}{ Altura $(\mathrm{cm})$} & \multirow{2}{*}{\multicolumn{2}{|c|}{$\begin{array}{c}\text { massa seca } \\
\text { (g/planta) } \\
\text { 60DAP }\end{array}$}} \\
\hline & & \multicolumn{2}{|l|}{ 20DAP } & \multicolumn{2}{|l|}{ 40DAP } & \multirow{2}{*}{$\begin{array}{c}\text { 60DAP } \\
\text { orig } \\
\end{array}$} & \multicolumn{2}{|l|}{ 20DAP } & \multicolumn{2}{|l|}{ 40DAP } & \multicolumn{2}{|l|}{ 60DAP } & \multicolumn{2}{|l|}{ 20DAP } & \multicolumn{2}{|l|}{ 40DAP } & \multicolumn{2}{|l|}{ 60DAP } & & \\
\hline & & orig & transf & orig & transf & & orig & transf & orig & $\operatorname{transf}$ & orig & $\operatorname{transf}$ & orig & $\operatorname{transf}$ & orig & $\operatorname{transf}$ & orig & transf & orig & $\operatorname{transf}$ \\
\hline & T1- Testemunha & 0,00 & $1,00 \mathrm{c}$ & 0,00 & $1,00 \mathrm{~b}$ & - & 37,25 & $6,18 \mathrm{a}$ & 31,50 & $5,69 \mathrm{a}$ & 29,00 & $5,47 \mathrm{a}$ & 11,25 & $3,5 \mathrm{a}$ & 15,25 & $4,0 \mathrm{a}$ & 10,50 & $3,39 \mathrm{a}$ & 6,00 & $2,64 \mathrm{a}$ \\
\hline & T2- imazapique $\left(245 \mathrm{~g} \mathrm{ha}^{-1}\right)$ & 10,00 & $3,32 \mathrm{~b}$ & 0,00 & $1,00 \mathrm{~b}$ & - & 33,75 & $5,89 \mathrm{a}$ & 31,25 & $5,66 \mathrm{a}$ & 33,00 & $5,82 \mathrm{a}$ & 11,00 & $3,5 \mathrm{a}$ & 13,75 & $3,8 \mathrm{a}$ & 14,00 & $3,87 \mathrm{a}$ & 7,31 & $2,87 \mathrm{a}$ \\
\hline $\bar{z}$ & T3- diclosulam $\left(88,2 \mathrm{~g} \mathrm{ha}^{-1}\right)+\mathrm{s}$-metolacloro $\left(1920 \mathrm{~g} \mathrm{ha}^{-1}\right)$ & 10,00 & $3,32 b$ & 0,00 & $1,00 \mathrm{~b}$ & - & 32,50 & $5,78 \mathrm{a}$ & 30,50 & $5,61 \mathrm{a}$ & 32,75 & $5,79 \mathrm{a}$ & 9,00 & $3,2 \mathrm{a}$ & 11,75 & $3,6 \mathrm{a}$ & 12,75 & $3,70 \mathrm{a}$ & 8,24 & $3,03 \mathrm{a}$ \\
\hline ర్ల & T4- imazapir $\left(500 \mathrm{~g} \mathrm{ha}^{-1}\right)$ & 26,25 & $5,20 \mathrm{a}$ & 9,75 & $3,28 \mathrm{a}$ & - & 33,00 & $5,83 \mathrm{a}$ & 27,25 & $5,28 \mathrm{a}$ & 30,25 & $5,59 \mathrm{a}$ & 11,25 & $3,5 \mathrm{a}$ & 12,25 & $3,6 \mathrm{a}$ & 13,25 & $3,78 \mathrm{a}$ & 5,79 & $2,60 \mathrm{a}$ \\
\hline 迅 & T5- diclosulam (88,2 g ha)+oxy fluorfen (1200 $\left.\mathrm{g} \mathrm{ha}^{-1}\right)$ & 0,00 & $1,00 \mathrm{c}$ & 0,00 & $1,00 \mathrm{~b}$ & - & 31,75 & $5,72 \mathrm{a}$ & 28,50 & $5,42 \mathrm{a}$ & 26,25 & $5,21 \mathrm{a}$ & 11,00 & $3,5 \mathrm{a}$ & 13,75 & $3,8 \mathrm{a}$ & 13,75 & $3,84 \mathrm{a}$ & 6,32 & $2,69 \mathrm{a}$ \\
\hline$\varangle$ & T6- amicarbazona $\left(2100 \mathrm{~g} \mathrm{ha}^{-1}\right)$ & 0,00 & $1,00 \mathrm{c}$ & 0,00 & $1,00 \mathrm{~b}$ & - & 31,00 & $5,65 \mathrm{a}$ & 32,50 & $5,79 \mathrm{a}$ & 35,00 & $5,99 \mathrm{a}$ & 12,00 & $3,6 \mathrm{a}$ & 13,50 & $3,8 \mathrm{a}$ & 12,50 & $3,64 \mathrm{a}$ & 4,86 & $2,40 \mathrm{a}$ \\
\hline 气 & T7-trifluralin $\left(2250 \mathrm{~g} \mathrm{ha}^{-1}\right)+$ pendimethalin $\left(1750 \mathrm{~g} \mathrm{ha}^{-1}\right)$ & 10,00 & $3,32 \mathrm{~b}$ & 0,00 & $1,00 \mathrm{~b}$ & - & 33,00 & $5,83 \mathrm{a}$ & 31,00 & $5,66 \mathrm{a}$ & 34,25 & $5,92 \mathrm{a}$ & 10,00 & $3,3 \mathrm{a}$ & 13,25 & $3,8 \mathrm{a}$ & 13,50 & $3,81 \mathrm{a}$ & 6,81 & $2,79 \mathrm{a}$ \\
\hline 0 & T8- sulfentrazona $\left(800 \mathrm{~g} \mathrm{ha}^{-1}\right)$ & 0,00 & $1,00 \mathrm{c}$ & 0,00 & $1,00 \mathrm{~b}$ & - & 30,50 & $5,60 \mathrm{a}$ & 27,75 & $5,36 \mathrm{a}$ & 32,00 & $5,74 \mathrm{a}$ & 17,75 & $3,4 \mathrm{a}$ & 13,00 & $3,7 \mathrm{a}$ & 12,25 & $3,59 \mathrm{a}$ & 7,14 & $2,82 \mathrm{a}$ \\
\hline & $\mathrm{CV}(\%)$ & - & 6,94 & - & 2,12 & - & - & 5,06 & - & 7,06 & - & 7,67 & - & 6,07 & - & 5,40 & - & 9,55 & - & 10,78 \\
\hline & $\mathrm{dms}$ & - & 0,39 & - & 0,07 & - & - & 0,69 & - & 0,92 & - & 1,02 & - & 0,48 & - & 0,48 & - & 0,83 & - & 0,69 \\
\hline & T1- Testemunha & - & - & - & - & - & 35,50 & $6,04 a$ & 30,00 & $5,55 \mathrm{a}$ & 25,00 & $5,09 \mathrm{a}$ & 11,25 & $3,48 \mathrm{a}$ & 12,75 & $3,70 \mathrm{a}$ & 12,75 & $3,70 \mathrm{a}$ & 4,55 & $2,34 \mathrm{a}$ \\
\hline & T2- imazapique $\left(245 \mathrm{~g} \mathrm{ha}^{-1}\right)$ & - & - & - & - & - & 32,50 & $5,79 \mathrm{a}$ & 30,25 & $5,57 \mathrm{a}$ & 30,25 & $5,57 \mathrm{a}$ & 12,75 & $3,71 \mathrm{a}$ & 12,75 & $3,70 \mathrm{a}$ & 13,50 & $3,81 \mathrm{a}$ & 4,03 & $2,23 \mathrm{a}$ \\
\hline$\widehat{\overbrace{}}$ & T3- diclosulam $\left(88,2 \mathrm{~g} \mathrm{ha}^{-1}\right)+\mathrm{s}$-metolacloro $\left(1920 \mathrm{~g} \mathrm{ha}^{-1}\right)$ & - & - & - & - & - & 29,75 & $5,54 \mathrm{a}$ & 26,50 & $5,23 \mathrm{a}$ & 22,50 & $4,85 \mathrm{a}$ & 9,50 & $3,24 \mathrm{a}$ & 10,50 & $3,39 \mathrm{a}$ & 10,75 & $3,42 \mathrm{a}$ & 3,17 & $2,04 \mathrm{a}$ \\
\hline ర్ల & T4- imazapir $\left(500 \mathrm{~g} \mathrm{ha}^{-1}\right)$ & - & - & - & - & - & 31,50 & $5,69 \mathrm{a}$ & 31,25 & $5,67 \mathrm{a}$ & 26,25 & $5,21 \mathrm{a}$ & 10,50 & $3,39 \mathrm{a}$ & 9,25 & $3,20 \mathrm{a}$ & 10,75 & $3,43 a$ & 3,26 & $2,06 \mathrm{a}$ \\
\hline 迅 & T5- diclosulam ( $88,2 \mathrm{~g}$ ha)+oxy fluorfen $\left(1200 \mathrm{~g} \mathrm{ha}^{-1}\right)$ & - & - & - & - & - & 30,75 & $5,63 \mathrm{a}$ & 35,50 & $6,03 \mathrm{a}$ & 27,75 & $5,36 \mathrm{a}$ & 11,25 & $3,50 \mathrm{a}$ & 11,25 & $3,49 \mathrm{a}$ & 11,75 & $3,57 \mathrm{a}$ & 3,98 & $2,22 \mathrm{a}$ \\
\hline 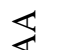 & T6- amicarbazona $\left(2100 \mathrm{~g} \mathrm{ha}^{-1}\right)$ & - & - & - & - & - & 29,00 & $5,47 \mathrm{a}$ & 28,25 & $5,40 \mathrm{a}$ & 28,75 & $5,45 \mathrm{a}$ & 11,00 & $3,46 \mathrm{a}$ & 12,00 & $3,60 \mathrm{a}$ & 12,50 & $3,66 \mathrm{a}$ & 3,66 & $2,16 \mathrm{a}$ \\
\hline 今 & T7-trifluralin $\left(2250 \mathrm{~g} \mathrm{ha}^{-1}\right)+$ pendimethalin $\left(1750 \mathrm{~g} \mathrm{ha}^{-1}\right)$ & - & - & - & - & - & 30,75 & $5,63 \mathrm{a}$ & 31,75 & $5,70 \mathrm{a}$ & 26,50 & $5,24 \mathrm{a}$ & 11,75 & $3,57 \mathrm{a}$ & 10,50 & $3,38 \mathrm{a}$ & 13,00 & $3,74 \mathrm{a}$ & 4,40 & $2,31 \mathrm{a}$ \\
\hline 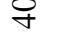 & T8- sulfentrazona $\left(800 \mathrm{~g} \mathrm{ha}^{-1}\right)$ & - & - & - & - & - & 29,00 & $5,48 \mathrm{a}$ & 36,25 & $6,10 \mathrm{a}$ & 28,00 & $5,38 \mathrm{a}$ & 11,75 & $3,57 \mathrm{a}$ & 12,25 & $3,63 a$ & 12,00 & $3,60 \mathrm{a}$ & 4,29 & $2,30 \mathrm{a}$ \\
\hline & $\mathrm{CV}(\%)$ & - & - & - & - & - & - & 5,59 & - & 8,06 & - & 6,24 & - & 6,25 & - & 7,01 & - & 7,09 & - & 9,01 \\
\hline & $\mathrm{dms}$ & - & - & - & - & - & - & 0,74 & - & 1,07 & - & 0,77 & - & 0,51 & - & 0,58 & - & 0,6 & - & 0,47 \\
\hline & T1- Testemunha & - & - & - & - & - & 29,00 & $5,47 \mathrm{a}$ & 30,00 & $5,51 \mathrm{a}$ & 32,25 & $5,73 \mathrm{a}$ & 12,50 & $3,67 \mathrm{a}$ & 15,00 & $3,99 \mathrm{a}$ & 15,25 & $4,03 a$ & 8,68 & $3,07 \mathrm{a}$ \\
\hline & T2- imazapique $\left(245 \mathrm{~g} \mathrm{ha}^{-1}\right)$ & - & - & - & - & - & 33,00 & $5,82 \mathrm{a}$ & 33,25 & $5,78 \mathrm{a}$ & 32,00 & $5,72 \mathrm{a}$ & 14,25 & $3,91 \mathrm{a}$ & 15,00 & $4,00 \mathrm{a}$ & 16,50 & $4,19 \mathrm{a}$ & 8,27 & $3,00 \mathrm{a}$ \\
\hline$\tilde{m}$ & T3- diclosulam $\left(88,2 \mathrm{~g} \mathrm{ha}^{-1}\right)+\mathrm{s}$-metolacloro $\left(1920 \mathrm{~g} \mathrm{ha}^{-1}\right)$ & - & - & - & - & - & 32,75 & $5,79 \mathrm{a}$ & 33,25 & $5,80 \mathrm{a}$ & 31,00 & $5,64 \mathrm{a}$ & 12,25 & $3,62 \mathrm{a}$ & 13,25 & $3,73 a$ & 13,75 & $3,82 \mathrm{a}$ & 9,44 & $3,16 \mathrm{a}$ \\
\hline ฮ్ & T4- imazapir $\left(500 \mathrm{~g} \mathrm{ha}^{-1}\right)$ & - & - & - & - & - & 30,25 & $5,58 \mathrm{a}$ & 32,25 & $5,70 \mathrm{a}$ & 31,75 & $5,70 \mathrm{a}$ & 12,25 & $3,62 \mathrm{a}$ & 13,50 & $3,79 \mathrm{a}$ & 14,25 & $3,90 \mathrm{a}$ & 7,36 & $2,88 \mathrm{a}$ \\
\hline 迅 & T5- diclosulam $\left(88,2 \mathrm{~g}\right.$ ha)+oxy fluorfen $\left(1200 \mathrm{~g} \mathrm{ha}^{-1}\right)$ & - & - & - & - & - & 26,25 & $5,22 \mathrm{a}$ & 31,50 & $5,65 \mathrm{a}$ & 33,25 & $5,83 \mathrm{a}$ & 10,75 & $3,42 \mathrm{a}$ & 11,00 & $3,46 \mathrm{a}$ & 12,50 & $3,67 \mathrm{a}$ & 6,04 & $2,62 \mathrm{a}$ \\
\hline$\varangle$ & T6- amicarbazona $\left(2100 \mathrm{~g} \mathrm{ha}^{-1}\right)$ & - & - & - & - & - & 35,00 & $5,99 \mathrm{a}$ & 33,50 & $5,83 \mathrm{a}$ & 30,50 & $5,59 \mathrm{a}$ & 13,00 & $3,74 \mathrm{a}$ & 13,25 & $3,77 \mathrm{a}$ & 13,50 & $3,81 \mathrm{a}$ & 7,89 & $2,96 \mathrm{a}$ \\
\hline$\stackrel{\hbar}{ٍ}$ & T7-trifluralin $\left(2250 \mathrm{~g} \mathrm{ha}^{-1}\right)+$ pendimethalin $\left(1750 \mathrm{~g} \mathrm{ha}^{-1}\right)$ & - & - & - & - & - & 34,25 & $5,92 \mathrm{a}$ & 32,25 & $5,74 \mathrm{a}$ & 32,75 & $5,78 \mathrm{a}$ & 13,00 & $3,74 \mathrm{a}$ & 14,25 & $3,90 \mathrm{a}$ & 14,75 & $3,96 \mathrm{a}$ & 9,13 & $3,14 \mathrm{a}$ \\
\hline 8 & T8- sulfentrazona $\left(800 \mathrm{~g} \mathrm{ha}^{-1}\right)$ & - & - & - & - & - & 32,00 & $5,74 \mathrm{a}$ & 31,50 & $5,68 \mathrm{a}$ & 30,75 & $5,60 \mathrm{a}$ & 11,75 & $3,57 \mathrm{a}$ & 13,75 & $3,84 \mathrm{a}$ & 12,25 & $3,64 \mathrm{a}$ & 6,16 & $2,64 \mathrm{a}$ \\
\hline & $\mathrm{CV}(\%)$ & - & - & - & - & - & - & 7,67 & - & 15,26 & - & 11,08 & - & 7,82 & - & 8,93 & - & 6,95 & - & 18,89 \\
\hline & $\mathrm{dms}$ & - & - & - & - & - & - & 1,02 & - & 2,04 & - & 1,48 & - & 0,67 & - & 0,8 & - & 0,63 & - & 1,30 \\
\hline
\end{tabular}

DAA = Dias após aplicação ; DAP = dias após plantio; orig = dados originais; transf = Dados tranformados em Raiz de X + alfa $(=1)$ dos herbicidas 
Tabela 3. Zimograma (perfil izoenzimático) de peroxidase e a-esterase nas MPBs de cana de açúcar aos 60 DAP de cada época.

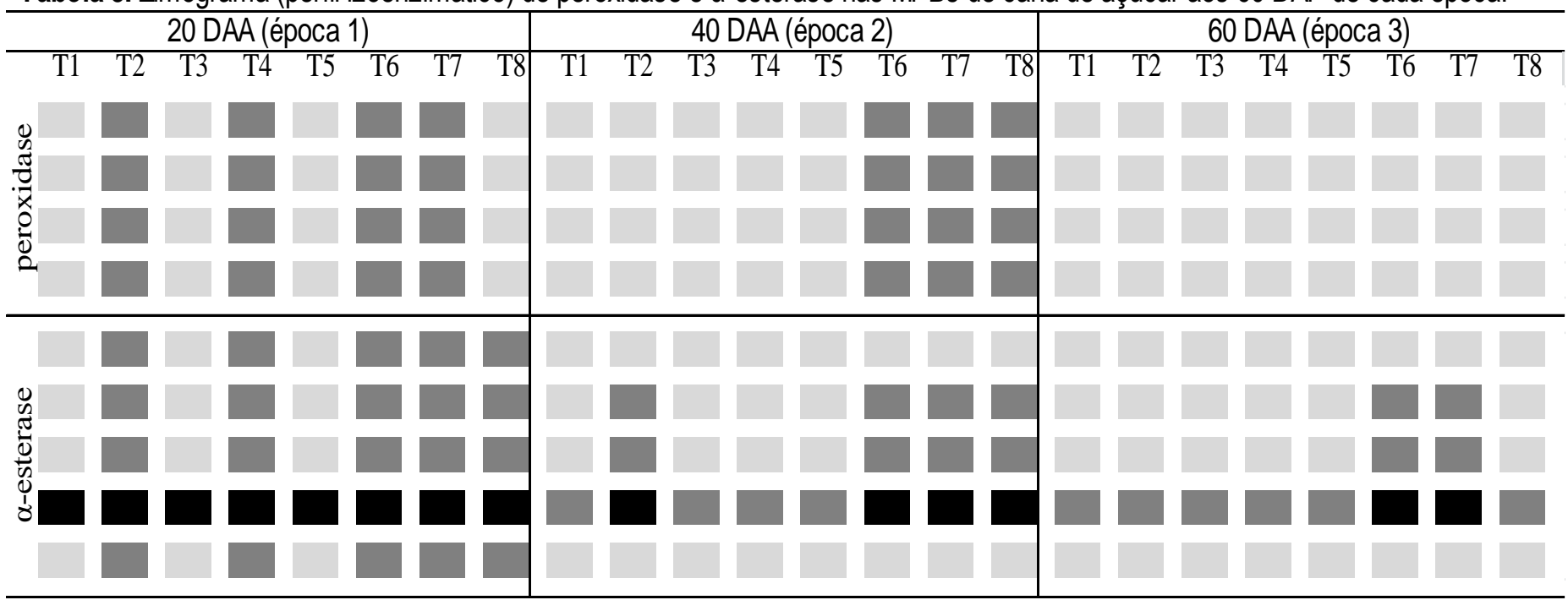

Tratamentos : T1- testemunha; T2- imazapique (245 g ha-1); T3- diclosulam(88,2 g ha-1) +s-metalacloro(1920g ha-1); T4- imazapir (500 g ha-1);T5- diclosulam (88,2 g ha-1)

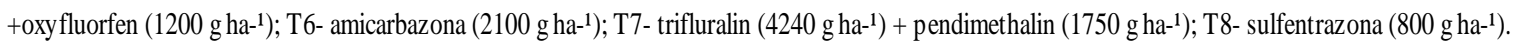

\section{CONCLUSÃO}

Objetivou-se estudar a aplicabilidade do perfil isoenzimático da $\alpha$-esterase e peroxidase, comparativamente às características fitotécnicas, como ferramenta para avaliar a seletividade na cultura da cana-de-açúcar, utilizando diversos herbicidas registrados em pré-plantio.

A hipótese inicial foi confirmada devido ao padrão das isoformas $\alpha$-esterase e peroxidase não diferirem em número de bandas, corroborando com as características fitotécnicas, evidenciando sintomas em determinados períodos inicias, e em períodos mais tardios apresentaram menos sintomas, mostrando uma recuperação fisiológica. Dessa forma verificou-se seletividade das MPBs de cana da cultivar IACSP95-5000. Assim, o perfil isoenzimático pode ser uma ferramenta para avaliação de seletividade de herbicidas quando associado às avaliações fitotécnicas.

\section{REFERÊNCIAS}

ABU-QARE, A. W.; DUNCAN, H. J. Herbicide safeners: uses, limitations, metabolism, and mechanisms of action. Chemosphere, v.48, p. 965-974, 2002.

ADRIANO, R.C.et al. Phenotypic and biochemical responses of sugarcane cultivars to glyphosate application. Sugar Tech, v.15, n.2 p.127-135, 2013.

ALFENAS, A.C.; BRUNE, W. Eletroforese em gel de amido. In: ALFENAS, A.C. (Ed). Eletroforese e marcadores bioquímicos em plantas e microrganismos. 2.ed. Viçosa: UFV, 2006. p.115 - 150b. 
ALFENAS, A.C.; BRUNE, W. Eletroforese em gel de poliacrilamida. In: ALFENAS, A.C. (Ed). Eletroforese e marcadores bioquímicos em plantas e microrganismos. 2.ed. Viçosa: UFV, 2006. p.151 - 182a.

ALFENAS, A.C.et al. Extração de proteínas para eletroforese. In: ALFENAS, A.C. (Ed). Eletroforese e marcadores bioquímicos em plantas e microrganismos. 2.ed. Viçosa: UFV, 2006. p. 85-114.

ALFENAS, A.C.et al. JUNGHANS, T.G. Eletroforese e marcadores bioquímicos em plantas e microrganismos. Viçosa: UFV, 2006. p.55-203.

ALMEIDA, M.; CRÓCOMO, O.J. Caracterização bioquímica de cultivares de cana- de- açúcar (Saccharum spp.): isoenzimas, proteínas solúveis e valor de brix. Scientia Agrícola, Piracicaba, v.51, n.3, p.422-429, 1994.

BELUCI, L. R.et al. Emergência e estabelecimento de plantas cultivadas após aplicação de glyphosate. Nucleus, v.11, n.1,p.141-152, 2014. Disponível em:

http://www.nucleus.feituverava.com.br/index.php/nucleus/article/view/977. DOI: 10.3738/1982.2278.977

BELUCI, L. R.et al. Resposta de mucuna preta (Mucuna Aterrima) aos herbicidas Amicarbazone e Imazapyr In: CONGRESSO INTERINSTITUCIONAL DE INICIAÇÃO CIENTÍFICA, 7. Anais... Campinas, 2013.

BEWLEY, J. D.; BLACK, M. Seed: physiology of development and germination. New York: Plenum Press, 1994. p.445.

BILANG, J.et al. A soluble auxin-binding protein from Hyoscyamus muticus is a glutathione s-transferase. Plant Physiology, v.102, p. 29-34, 1993.

BREAUX, E. J.; PATANELLA, J.E.; SANDERS, E. F. Chloroacetanilide Herbicide Selectivity: Analysis of Glutathione and Homoglutathione in Tolerant, Susceptible, and Safened Seedling. Journal of Agricultural and Food Chemistry, v.35, p.474-478, 1987.

DIONISIO-SESE, M.L.; TOBITA, S. Antioxidant responses of rice seedlings salinity stress. Plant Science, Strasbourg, v.135, p.1-9, 1998.

LOPES, J.P. et al. Análise de crescimento e trocas gasosas na cultura de milho em plantio direto e convencional. Bragantia, v. 68, p. 839-848, 2009.

MACHADO, R. F.et al. Reflexos do mecanismo de ação de herbicidas na qualidade fisiológica de sementes e na atividade enzimática em plântulas de arroz. Revista Brasileira de Sementes, v. 28, n.3, p.151-160, 2006.

MITTLER, R. Oxidative stress, antioxidants and stress tolerance. Trends in Plant Science, London, v.7, n.9, p. 405-410, Sept. 2002.

NEGRISOLI, E.et al. Seletividade de herbicidas aplicados em pré-emergência na cultura de cana-de-açúcar tratada com nematicidas. Planta Daninha, Viçosa, v.22, n. 4, p. 567-575, 2004.

SCANDÁLIOS, J.G. Genetic control of multiple molecular forms of enzymes in plants: a review. Biochemical Genetics, v.3, n.1, p.37-79, 1969.

SILVA, D.M.et al. Seletividade de herbicidas influenciada pelo estado nutricional da cana de açúcar. Revista Brasileira de Herbicidas, v.12, n.1, p.56-67, 2013. 
SREENIVASUTU, N.et al. Total peroxidase activity and peroxidase isoforms as modified by salt stress in two cultivars of fox-tail millet with differential salt tolerance. Plant Science, Strasbourg, v.141, p.1-9, 1999.

ULISSES, C.et al. Caracterização isoenzimática de clones de bananeira nanicão submetidos a salinidade. Revista Brasileira de Engenharia Agrícola e Ambiental, Campina Grande, v.6, n.2, p.358-361, 2002.

USHIMARU, T.et al. Antioxidative enzymes in seedlling of Nelumbo nucifera germinated under water. Physiology Plantarum, v.112, n.1, p.39-46, 2001.

VITORINO, R.. Controle químico de mucuna preta (Mucuna aterrima)com amicarbazone e imazapyr e a seletividade sobre a cana-de-açúcar. Ituverava: FE/FAFRAM, 2013. 41p. Trabalho de conclusão de curso (Graduação - Engenheiro Agrônomo). Faculdade Dr. Francisco Maeda. Fundação Educacional de Ituverava.

WALKER, C. H.; MACKNESS, M. I. Esterases: problems of identification and classification. Biochem. Pharmacol., v. 32, p. 3265-3269, 1983. 